\title{
On the MAC for Power-Line Communications: Modeling Assumptions and Performance Tradeoffs
}

\author{
Christina Vlachou*, Albert Banchs ${ }^{\dagger}$, Julien Herzen*, Patrick Thiran* \\ ${ }^{*}$ EPFL, Switzerland ${ }^{\dagger}$ Institute IMDEA Networks and University Carlos III of Madrid, Spain \\ *firstname.lastname@epfl.ch † banchs@it.uc3m.es
}

\begin{abstract}
Power-line communications are becoming a key component in home networking. The dominant MAC protocol for high data-rate power-line communications, IEEE 1901, employs a CSMA/CA mechanism similar to the backoff process of 802.11. Existing performance evaluation studies of this protocol assume that the backoff processes of the stations are independent (the so-called decoupling assumption). However, in contrast to 802.11, 1901 stations can change their state after sensing the medium busy, which introduces strong coupling between the stations and, as a result, makes existing analyses inaccurate.

In this paper, we propose a new performance model for $\mathbf{1 9 0 1}$, which does not rely on the decoupling assumption. We prove that our model admits a unique solution. We confirm the accuracy of our model using both testbed experiments and simulations, and we show that it surpasses current models based on the decoupling assumption. Furthermore, we study the tradeoff between delay and throughput existing with 1901. We show that this protocol can be configured to accommodate different throughput and jitter requirements, and we give systematic guidelines for its configuration.
\end{abstract}

\section{INTRODUCTION}

Power-line communications (PLC) are increasingly important in home networking. HomePlug AV, the most popular specification for PLC, is employed by over 120 million devices worldwide [1], and the new HomePlug AV2 devices offer data rates up to $1 \mathrm{Gbps}$ [2]. Moreover, PLC plays a powerful role in hybrid networks comprising wireless, Ethernet, and other technologies [3], as it contributes to increasing the bandwidth of such networks with an independent, widely accessible medium. Yet, despite the wide adoption of HomePlug specifications in home networks, little attention has been paid to providing an accurate analysis and an evaluation of the HomePlug MAC layer.

The vast majority of HomePlug devices employ a multipleaccess protocol based on CSMA/CA that is specified by the IEEE 1901 standard $^{1}$ [4]. This CSMA/CA mechanism resembles the CSMA/CA mechanism employed by IEEE 802.11, but with important differences in terms of complexity, performance and fairness. The main difference stems from the introduction of a so-called deferral counter that triggers a redraw of the backoff counter when a station senses the medium busy. This additional counter significantly increases the statespace required to describe the backoff procedure. Moreover, as we explain in more details later, the use of the deferral counter introduces some level of coupling between the stations, which

\footnotetext{
${ }^{1}$ This CSMA/CA mechanism is the same for all HomePlug specifications, including 1.0, AV, AV2 and GreenPhy.
}

978-1-4799-6204-4/14/\$31.00 (C)2014 IEEE penalizes the accuracy of models based on the decoupling assumption. This assumption was originally proposed in the 802.11 analysis of [5] and has been used in all the works that have analyzed the 1901 CSMA/CA procedure so far (i.e., [6], [7], [8]). In this paper, we show that this decoupling assumption leads to inaccurate results, and the modeling accuracy can be substantially improved by avoiding it.

The decoupling assumption relies on the approximation that the backoff processes of the stations are independent and that, as a consequence, stations experience the same time-invariant collision probability, independently of their own state and of the state of the other stations [5]. In addition, to analyze 1901, it has been assumed that a station senses the medium busy with the same time-invariant probability (equal to the collision probability), during any time slot [6], [7]. In this paper, we show that the deferral counter introduces some coupling among the stations: After a station gains access to the medium, it can retain it for many consecutive transmissions before any other station can transmit. As a result, the collision and busy probabilities are not time-invariant for 1901 networks, which makes the decoupling assumption questionable.

Figures 1 and 2 provide some evidence on the coupling phenomenon described above, for a HomePlug AV testbed with two stations. While Station $A$ transmits during several consecutive slots, Station $B$ is likely to remain in a state where it has a larger probability of colliding or sensing the medium busy. $B$ is then even less likely to attempt a transmission while in this state, and it might have to wait several tens of milliseconds before the situation reverts. Thus, the collision probabilities observed by the stations are clearly time-varying, which invalidates the decoupling assumption. Note that a consequence of this coupling is short-term unfairness, which in turn translates into high delay variance (i.e., high jitter).

In this paper, we propose a theoretical framework for modeling the CSMA/CA process of 1901 without relying on the decoupling assumption. First, we introduce a model that accurately captures the 1901 performance without assuming that stations are decoupled. This model is relatively compact: computing the throughput of the network only requires solving a system of $m$ equations, where $m$ is the number of backoff stages (the default value for 1901 is $m=4$ ). Second, we prove that this system of equations admits a unique solution. We confirm the accuracy of the model by using both simulations and a testbed of 7 HomePlug AV stations. To the best of our knowledge, this is the first study that validates a 1901 

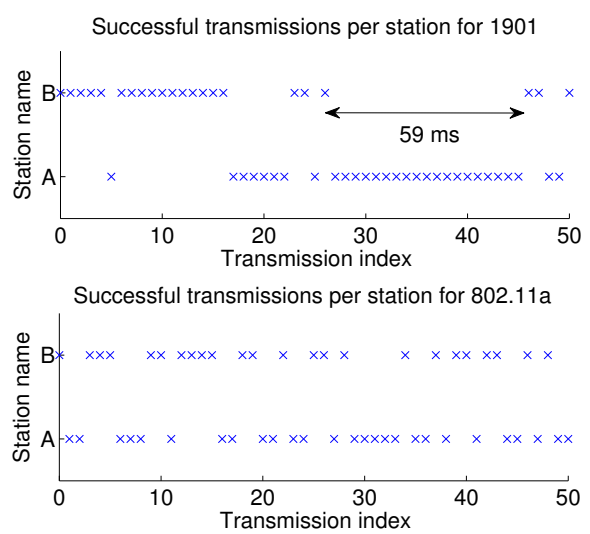

Fig. 1. Testbed trace of 50 successful transmissions by two saturated stations with 1901 and 802.11a. The experimental settings and the details on our testbed are given in Section V-A. 1901 exhibits short-term unfairness: a station holding the channel is likely to keep holding it for many consecutive transmissions (during several tens of ms, for example $59 \mathrm{~ms}$ as shown above), which causes high jitter. 802.11 is fairer, which makes the decoupling assumption viable in this case. This experiment took place under ideal channel conditions. In realistic channels, where frames are retransmitted due to errors, the jitter might even be higher.
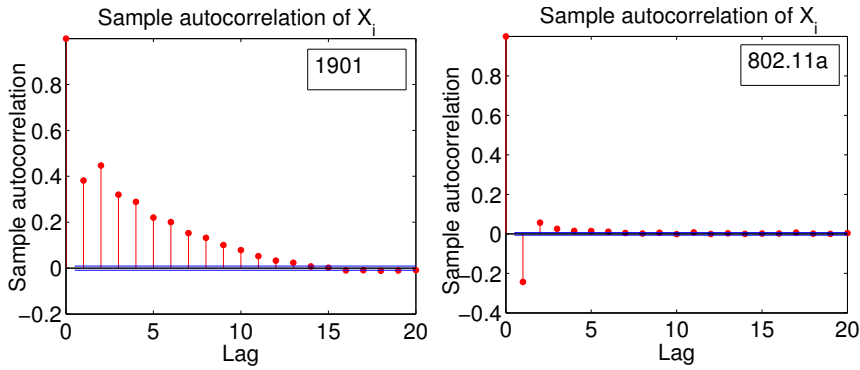

Fig. 2. We study a testbed trace of $5 \cdot 10^{4}$ successful transmissions for both 1901 and 802.11a when two saturated stations $A$ and $B$ contend for the medium Let $X_{i}$ be the variable that indicates which station transmits successfully at the $i$-th transmission. We take $X_{i}:=1$ if $A$ transmits and $X_{i}:=2$ if $B$ transmits. We show the autocorrelation function of $X_{i}, 1 \leq i \leq 5 \cdot 10^{4}$. Observe that it is positive for 1901 at lags smaller than 15. Hence, if $X_{i}=1$ for some $i$, it is likely that $X_{i+1}=1$. For 802.11a, we have in contrast a negative value of autocorrelation at lag 1 and a positive one at lag 2 , which means that if $X_{i}=1$ for some $i$, it is likely that $X_{i+1}=2$ and $X_{i+2}=1$.

MAC performance model on a real PLC testbed. We have employed a similar testbed setup to study and validate the short-term unfairness (but not a complete model) of the 1901 MAC in [9]. In addition, we investigate the accuracy of our model and that of previous works that rely on the decoupling assumption, showing that, to the best of our knowledge, ours is the first model for 1901 reaching this level of accuracy. Finally, we study in detail the tradeoff between throughput and delay variance (or equivalently, short-term fairness), caused by the deferral counter in 1901. We observe that this tradeoff can be accurately controlled in 1901, and propose a systematic method to configure a 1901 network to obtain the best delay performance under arbitrary throughput constraints.

The remainder of the paper is organized as follows. We present the 1901 backoff process in Section II. We then review the related work on MAC layer in Section III. We present our model for 1901 in Section IV. We evaluate the performance of our model and discuss the decoupling assumption in Section V. We study the tradeoff between throughput and fairness in Section VI. Finally, we give concluding remarks in Section VII.

\section{BACKGROUND}

In this section, we present the CSMA/CA protocol of 1901 [4], and highlight the mechanism that causes the strong coupling between the stations. This mechanism is the main difference between 1901 and 802.11. In CSMA/CA protocols, such as 802.11 , stations wait for a random number of time slots (determined by the backoff counter) before transmitting, in the attempt to avoid that some other station transmits at the same slot, which causes a collision. Nevertheless, a collision can still occur and when it does, the stations involved increase the range in which they select their backoff counter (called the contention window $C W$ ) to further reduce the collision probability. Clearly, there exists a tradeoff: if $C W$ is large, the collision probability is small, but under lowload conditions stations waste many slots on average before transmitting, which decreases throughput. As we explain later, to tackle this backoff inefficiency, 1901 aims at reducing $C W$. To counterbalance the resulting large collision probability, 1901 introduces an additional mechanism that increases $C W$ before a collision occurs: when a station senses a considerable number of transmissions in the channel, it increases $C W$. To count the number of times a station has to sense the medium busy before increasing $C W$, a new counter is introduced, called the deferral counter.

We now describe the technical details of the 1901 CSMA/CA procedure. It includes three counters: the backoff counter $(B C)$, the deferral counter $(D C)$ and the backoff procedure counter $(B P C)$. Upon the arrival of a new packet, a transmitting station enters backoff stage 0 . It then draws the backoff counter $B C$ uniformly at random in $\left\{0, \ldots, C W_{0}-1\right\}$, where $C W_{0}$ denotes the contention window used at backoff stage 0 . Similarly to $802.11, B C$ is decreased by 1 at each time slot if the station senses the medium to be idle (i.e., below the carrier-sensing threshold), and it is frozen when the medium is sensed busy. In the case the medium is sensed busy, $B C$ is also decreased by 1 once the medium is sensed idle again. When $B C$ reaches 0 , the station attempts to transmit the packet. Also similarly to 802.11, the station jumps to the next backoff stage if the transmission fails. In this case, the station increments the $B P C$ counter and enters the next backoff stage. The station then draws $B C$ uniformly at random in $\left\{0, \ldots, C W_{i}-1\right\}$, where $C W_{i}$ is the contention window used for backoff stage $i$, and repeats the process. For 802.11, the contention window is doubled between the successive backoff stages, i.e., $C W_{i}=2^{i} C W_{0}$. For 1901, $C W_{i}$ depends on the value of the $B P C$ counter and the priority of the packet: There are four backoff stages that are mapped to the $B P C$ counter, as given in Table I. Also, there are four different priority classes (CA0 to $\mathrm{CA} 3$ ) that correspond to different values for the $C W_{i}$ 's.

The main difference between 1901 and 802.11 is that a 1901 station might enter the next backoff stage even if it did not attempt a transmission. This is regulated by the deferral counter $D C$, which works as follows. When the station enters backoff stage $i, D C$ is set at an an initial DC value $d_{i}$, where $d_{i}$ is given in Table I for each backoff stage $i$. After having sensed 


\begin{tabular}{|c|c|c|c|c|c|c|c|}
\hline & tation & & & & Station & $B$ & \\
\hline $\begin{array}{l}\text { backoff } \\
\text { stage } i\end{array}$ & $C W_{i}$ & $D C$ & $B C$ & $\begin{array}{l}\text { backoff } \\
\text { stage } i\end{array}$ & $C W_{i}$ & $D C$ & $B C$ \\
\hline \multirow{9}{*}{$i=0$} & 8 & 0 & 3 & \multirow{3}{*}{$i=0$} & 8 & 0 & 5 \\
\hline & 8 & 0 & 0 & & & 0 & 2 \\
\hline & \multicolumn{3}{|c|}{ Transmission } & & & & \\
\hline & 8 & 0 & 7 & \multirow{6}{*}{$i=1$} & 16 & 1 & 11 \\
\hline & & & & & & & \\
\hline & 8 & 0 & $\mathbf{0}$ & & 16 & 1 & 4 \\
\hline & \multicolumn{3}{|c|}{ Transmission } & & & & \\
\hline & 8 & 0 & 5 & & 16 & 0 & 3 \\
\hline & \multirow[t]{2}{*}{8} & 0 & 2 & & 16 & 0 & 0 \\
\hline & & & & & \multicolumn{3}{|c|}{ Transmission } \\
\hline$i=1$ & 16 & 1 & 6 & $i=0$ & 8 & 0 & 2 \\
\hline
\end{tabular}

Fig. 3. An example of the time evolution of the 1901 backoff process with 2 saturated stations $A$ and $B$. Initially, both stations start at backoff stage 0 . Station $A$ wins the channel for two consecutive transmissions. Observe the change in $C W_{i}$ when a station senses the medium busy and has $D C=0$. This figure also exposes the short-term unfairness when there are 2 contending stations; a station that grabs the channel for a successful transmission moves to backoff stage 0 , whereas the other station enters a higher backoff stage with larger $C W$ and has lower probability to transmit.

the medium busy, a station decreases $D C$ by 1 (in addition to $B C$ ). If the medium is sensed busy and $D C=0$, then the station jumps to the next backoff stage (or re-enters the last backoff stage, if it is already at this stage) and re-draws $B C$, without attempting a transmission. An example of such a backoff process is shown in Figure 3.

\begin{tabular}{cc|cc|cc} 
& \multicolumn{3}{c}{ Class CA0/CA1 } & \multicolumn{2}{c}{ Class CA2/CA3 } \\
\hline \hline backoff stage $i$ & $B P C$ & $C W_{i}$ & $d_{i}$ & $C W_{i}$ & $d_{i}$ \\
0 & 0 & 8 & 0 & 8 & 0 \\
1 & 1 & 16 & 1 & 16 & 1 \\
2 & 2 & 32 & 3 & 16 & 3 \\
3 & $\geq 3$ & 64 & 15 & 32 & 15 \\
\hline
\end{tabular}

TABLE I

IEEE 1901 VALUES FOR THE CONTENTION WINDOWS $C W_{i}$ AND THE INITIAL VALUES $d_{i}$ OF DEFERRAL COUNTER $D C$, FOR EACH BACKOFF STAGE $i$ AND EACH PRIORITY CLASS. CA0/CA1 PRIORITIES ARE USED FOR BEST-EFFORT TRAFFIC AND CA2/CA3 FOR DELAY-SENSITIVE TRAFFIC.

The deferral counter was introduced in 1901, so that 1901 can employ small contention window values - which provide good performance for a small number of stations - while avoiding collisions, thus also providing good performance for a large number of stations ${ }^{2}$. In particular, to reduce collisions, 1901 stations redraw their backoff counter when they sense a number of transmissions before their backoff counter expires; in this way, they can react to a high load in the network without the need of a collision, which is in contrast to 802.11 that only reacts to collisions.

Although the above mechanism achieves its goal, i.e., providing good performance in terms of throughput, it might lead to short-term unfairness: When a station gets hold of the channel and uses a small contention window, it is likely to transmit several frames and thus trigger the deferral counter mechanism of the other stations, which further increase their contention windows and hence reduce even more their probability of

\footnotetext{
${ }^{2}$ Indeed, it can be seen from Table I that 1901 contention windows are small.
}

accessing the channel. Such a coupling effect penalizes the accuracy of existing models that assume that the backoff process of different stations are independent. Furthermore, another consequence of this behavior is that a station either holds the channel, and thus experiences low delays, or has to wait a long time before it can transmit. This causes high jitter (i.e., high delay variance).

\section{RELATED WORK}

The backoff process of 802.11 can be considered as a version of 1901 where the deferral counter $D C$ never reaches 0 (i.e., $d_{i}=\infty$, for all $i$ ). Hence, in the following, we first review relevant studies on 802.11, both with and without the decoupling assumption, and then we present the existing work on 1901. Finally, we discuss related works on fairness.

\section{A. Analyses of IEEE 802.11}

Most work modeling 802.11 performance relies on the decoupling assumption, initially proposed by Bianchi in [5]. In his paper, Bianchi proposes a model for single contention domains, using a discrete-time Markov chain to model the backoff procedure of 802.11. Under the decoupling assumption, the collision probability experienced by all stations is time-invariant and can be found via a fixed-point equation that depends on the parameters of the protocol. Kumar et al. [10] examine the backoff process of 802.11 using the same assumptions and renewal theory. The authors also extract a fixed-point equation for the collision probability. Although strong, the decoupling assumption has later been examined and found to be a valid assumption for 802.11 (shown analytically and experimentally in [11], [12], respectively).

Sharma et al. [13] study 802.11 without the decoupling assumption. They analyze an $m$-dimensional chain ( $m$ being the number of backoff stages) that describes the number of stations at each backoff stage. Drift equations capture the expected change on the number of stations at each backoff stage between two consecutive time slots, and their equilibrium point yields the average number of stations at each backoff stage in steady state. Similarly to [13], we also use drift equations to obtain an accurate model for 1901, without resorting to the decoupling assumption. However, as the 1901 protocol is much more complex than 802.11 , so is our analysis; it differs substantially from the one of [13].

\section{B. Analyses of IEEE 1901 under the Decoupling Assumption}

To the best of our knowledge, the only works analyzing the backoff mechanism of 1901 rely on the decoupling assumption. First, Chung et al. [6] introduce a model using a discretetime Markov chain similar to Bianchi's model for 802.11 [5]. The additional state required to capture the effect of the deferral counter $D C$ significantly increases the complexity of the Markov chain.

Second, we [7] propose a simplification of the Markov chain [6] and an equivalent model in the form of a single fixed-point equation. We apply the same theoretical framework as [10] and prove that this equation admits a unique solution. 
Finally, Cano and Malone [8] provide a simplification of the analysis of [6] for computing the delay under unsaturated traffic scenarios and discuss the assumptions used in [6]. Here too, their analysis relies on the decoupling assumption.

\section{MAC Layer Short-Term Fairness}

Various investigations evaluating short-term fairness of MAC protocols have been conducted. First, Berger-Sabbatel et al. [14] study the 802.11 short-term fairness both analytically and experimentally. They prove that the 802.11 MAC is short-term fair when there are few contending stations. Second, Bredel and Fidler [15] elaborate more on the 802.11 backoff process and investigate fairness both in short-term and long-term.

Finally, we explore the 1901 fairness both analytically and experimentally using simulation and a testbed in [9]. We reveal that, compared to $802.11,1901$ is short-term unfair, particularly when there are 2 stations contending for the medium.

\section{ANALYSIS}

In this section, we introduce our model for the 1901 CSMA/CA protocol. Our analysis relies on the following assumptions:

- There are $N$ stations that belong to a single contention domain.

- All stations are saturated (always have a packet to send).

- There is no packet loss or errors due to the physical layer, and transmission failures are only due to collisions.

- The stations have an infinite retry limit; that is, they never discard a packet until it is successfully transmitted ${ }^{3}$.

The 1901 standard introduces four different priority classes (see Section II) and specifies that only the stations belonging to the highest contending priority class run the backoff process ${ }^{4}$. In our analysis, we follow this property, and we consider a scenario in which all the contending stations use the same set of parameters (corresponding to the highest priority class).

Our model is a dynamical system that describes the expected change in the number of stations at each backoff stage between any two consecutive time slots. In the stationary regime, the expected number of stations at each backoff stage is constant, hence we can compute performance metrics by finding the equilibrium of the dynamical system.

Let us now introduce the variables of our model. Let $m$ be the number of backoff stages and let $n_{i}, 0 \leq i \leq m-1$ denote the number of stations at backoff stage $i$. Note that $\sum_{i=0}^{m-1} n_{i}=N$. Let us further denote with $\tau_{i}$ the transmission probability at stage $i$, i.e., $\tau_{i}$ is the probability that a station at backoff stage $i$ transmits at any given time slot. In addition, for a given station at backoff stage $i$, we denote with $p_{i}$ the probability that at least one other station transmits. We also denote with $p_{e}$ the

\footnotetext{
${ }^{3}$ Contrary to 802.11 , the 1901 standard does not specify a retry limit. However, there is a timeout on the frame transmission that is vendor specific. For instance, for the HomePlug AV devices tested in Section V, the timeout for CA1 priority frames is $2.5 \mathrm{~s}$, which is very large compared to the maximum frame duration (2.5 ms [4]). Therefore, the infinite retry limit assumption is not strong.

${ }^{4}$ In practice, the contending priority class is decided during a so-called priority resolution phase, using a simple system of busy tones.
}

probability that no station transmits (or equivalently, that the medium is idle). Under the assumption of independence of the transmission attempts in a single contention domain, we have $p_{e}=\prod_{k=0}^{m-1}\left(1-\tau_{k}\right)^{n_{k}}$, and therefore

$$
p_{i}=1-\frac{p_{e}}{1-\tau_{i}}=1-\frac{1}{1-\tau_{i}} \prod_{k=0}^{m-1}\left(1-\tau_{k}\right)^{n_{k}} .
$$

In 1901, a station with $D C$ equal to $d_{i}$ can change its backoff stage either (i) after attempting a transmission or (ii) due to sensing the medium busy $d_{i}+1$ times. ${ }^{5}$ To compute the probabilities of events (i) and (ii), we introduce $x_{k}^{i}$ : it is the probability that a station at backoff stage $i$ jumps to the next backoff stage $i+1$ in $k$ or fewer time slots due to (ii). Note that we can compute $x_{k}^{i}$ directly from $p_{i}$. Let $T$ be the random variable describing the number of slots among $k$ slots during which the medium is sensed busy. Because a station at backoff stage $i$ senses the medium busy with probability $p_{i}$ at each time slot, $T$ follows the binomial distribution $\operatorname{Bin}\left(k, p_{i}\right)$. This yields

$$
x_{k}^{i}=\mathbb{P}\left(T>d_{i}\right)=\sum_{j=d_{i}+1}^{k}\left(\begin{array}{l}
k \\
j
\end{array}\right) p_{i}^{j}\left(1-p_{i}\right)^{k-j} .
$$

Let us denote with $b c_{i}$ the expected number of time slots spent by a station at backoff stage $i$. Now, recall that when entering stage $i$, the stations draw a backoff counter $B C$ uniformly at random in $\left\{0, \ldots, C W_{i}-1\right\}$. Let $k$ denote the value of $B C$, and $d_{i}$ be the value of $D C$ when the station enters stage $i$. Depending on $k$, one of the following happens:

- If $k>d_{i}$, then event (i) occurs with probability $\left(1-x_{k}^{i}\right)$, in which case the station spends $(k+1)$ slots in stage $i$ (the $(k+1) t h$ slot being used for transmission). This event is illustrated by the two transmissions of station A in Figure 3. Now, (ii) occurs with probability $x_{k}^{i}$. More precisely, (ii) occurs at slot $j$, for $d_{i}+1 \leq j \leq k$, with probability $\left(x_{j}^{i}-x_{j-1}^{i}\right)^{6}$, in which case the station spends $j$ slots in stage $i$.

- If $k \leq d_{i}$, then (ii) cannot happen. Event (i) takes place with probability 1 , which yields that the backoff counter expires and that the station spends $(k+1)$ slots in stage $i$.

By grouping all the possible cases described above, it follows that $b c_{i}$ is given by

$$
\begin{aligned}
b c_{i} & =\frac{1}{C W_{i}} \sum_{k=d_{i}+1}^{C W_{i}-1}\left[(k+1)\left(1-x_{k}^{i}\right)+\sum_{j=d_{i}+1}^{k} j\left(x_{j}^{i}-x_{j-1}^{i}\right)\right] \\
& +\frac{\left(d_{i}+1\right)\left(d_{i}+2\right)}{2 C W_{i}} .
\end{aligned}
$$

Now, the transmission probability $\tau_{i}$ can be expressed as a function of $x_{k}^{i}$ and $b c_{i}$, using the renewal-reward theorem, with the number of backoff slots spent in stage $i$ being the

\footnotetext{
${ }^{5}$ A major difference between 1901 and 802.11 is that, contrary to 1901 , a station using 802.11 can only adapt its backoff because of (i), not of (ii).

${ }^{6}$ Observe that $\left(x_{j}^{i}-x_{j-1}^{i}\right)$ is the difference of two complementary CDFs and denotes the probability that (ii) happens exactly at slot $j$.
} 
renewal sequence and the number of transmission attempts (i.e., 0 or 1) being the reward. The expected number of transmission attempts at stage $i$ can be computed similarly to $b c_{i}$. Hence, by dividing the expected transmission attempts at stage $i$ with the expected time slots spent at stage $i, \tau_{i}$ is given by

$$
\tau_{i}=\frac{\sum_{k=d_{i}+1}^{C W_{i}-1} \frac{1}{C W_{i}}\left(1-x_{k}^{i}\right)+\frac{d_{i}+1}{C W_{i}}}{b c_{i}} .
$$

Similarly, we define $\beta_{i}$ as the probability that, at any given slot, a station at stage $i$ moves to the next backoff stage because it has sensed the medium busy $d_{i}+1$ times. It can be easily seen that $\beta_{i}$ is given by

$$
\beta_{i}=\frac{\sum_{k=d_{i}+1}^{C W_{i}-1} \frac{1}{C W_{i}} \sum_{j=d_{i}+1}^{k}\left(x_{j}^{i}-x_{j-1}^{i}\right)}{b c_{i}} .
$$

Note that $\tau_{i}$ and $\beta_{i}$ are functions of $p_{i}$ (through $x_{k}^{i}$ and $b c_{i}$ ).

We next introduce our model. A key feature of our model is that we do not assume that the stations are decoupled, as the collision probability is allowed to depend on the station's state. To study the system, we use a vector that includes the number of stations at each backoff stage. In particular, let $X(t)=\left(X_{0}(t), X_{1}(t), \ldots, X_{m-1}(t)\right)$ represent the number of stations at each backoff stage $(0,1, \ldots, m-1)$ at time slot $t$. We use the notation $n(t)=\left(n_{0}(t), n_{1}(t), \ldots, n_{m-1}(t)\right)$ to denote a realization of $X(t)$ at some time slot $t$.

To analyze our system, we assume that the backoff counters are geometrically distributed with the same mean of that of the real uniform distribution. With this assumption, a station at backoff stage $i$ transmits at any slot $t$ with a constant probability $\tau_{i}$ given by (4), independently from the previous slots, and the vector $X(t)$ is a Markov chain. Furthermore, we assume that at backoff stage $i$ a station might move to the next backoff stage due to sensing the medium busy with probability $\beta_{i}$. Note that $p_{i}, \tau_{i}$, and $\beta_{i}$ can be computed from (1), (4) and (5), given the state vector $n(t)$ (hereafter, to simplify notation we drop the input variable $t$ from $p_{i}(t), \tau_{i}(t), \beta_{i}(t)$, and $n(t)$ as the equations are expressed for any slot $t)$.

Let now $F(n)=\mathbb{E}[X(t+1)-X(t) \mid X(t)=n]$ be the expected change in $X(t)$ over one time slot, given that the system is at state $n$. The function $F(\cdot)$ is called the drift of the system, and is given by

$$
\begin{aligned}
& F_{i}(n)= \\
& \left\{\begin{array}{l}
\sum_{k=0}^{m-1} n_{k} \tau_{k}\left(1-p_{k}\right)-n_{0}\left(\tau_{0}+\beta_{0}\right), \quad i=0 \\
n_{i-1}\left(\tau_{i-1} p_{i-1}+\beta_{i-1}\right)-n_{i}\left(\tau_{i}+\beta_{i}\right), 0<i<m-1 \\
n_{m-2}\left(\tau_{m-2} p_{m-2}+\beta_{m-2}\right)-n_{m-1} \tau_{m-1}\left(1-p_{m-1}\right), \\
i=m-1 .
\end{array}\right.
\end{aligned}
$$

(DRIFT) is obtained by balancing, for every backoff stage, the average rate of stations that enter and leave this backoff stage. In particular, $F_{0}$ increases only when some stations transmit successfully. Since such stations could be in any of the other backoff stages and there are $n_{k}$ stations in stage $k$, this occurs at rate $\sum_{k=1}^{m-1} n_{k} \tau_{k}\left(1-p_{k}\right)$. Similarly, $F_{0}$ decreases when some stations at stage 0 are either involved in a collision (which occurs with probability $\left.n_{0} \tau_{0} p_{0}\right)$, or do not transmit and sense the medium busy $d_{0}+1$ times (which occurs with probability $\left.n_{0} \beta_{0}\right)$. The decrease of the drift in both cases is 1 , thus the expected decrease is equal to the sum of the two probabilities.

Similarly, $F_{i}, 0<i<m-1$ is computed by observing that in these backoff stages, $F_{i}$ changes if and only if some stations

\begin{tabular}{|c|c|}
\hline$n_{i}$ & Number of stations \\
\hline$p_{i}$ & Probability that at least one other station transmits at any slot \\
\hline$p_{e}$ & Probability that the medium is idle at any slot (same for all $i$ ) \\
\hline$x_{k}^{i}$ & $\begin{array}{l}\text { Probability that a station leaves stage } i \text { due to sensing the } \\
\text { medium busy } d_{i}+1 \text { times (the backoff counter does not expire) }\end{array}$ \\
\hline$b c_{i}$ & Expected number of backoff slots \\
\hline$\tau_{i}$ & Probability that a station transmits at any slot \\
\hline$\beta_{i}$ & $\begin{array}{l}\text { Probability that a station leaves stage } i \text { due to sensing the } \\
\text { medium busy } d_{i}+1 \text { times at any slot }\end{array}$ \\
\hline$F_{i}$ & Expected change in $n_{i}$ between two consecutive slots \\
\hline $\bar{n}_{i}$ & Expected number of stations \\
\hline
\end{tabular}
sense the medium busy, or transmit. Finally, $F_{m-1}$ increases after some stations at stage $m-2$ experience a collision or sense the medium busy $d_{m-2}+1$ times. It decreases only after a successful transmission at stage $m-1$.

The evolution of the expected number of stations $\bar{n}(t):=$ $\mathbb{E}[X(t)]$ is described by the $m$-dimensional dynamical system

$$
\bar{n}(t+1)=\bar{n}(t)+F(\bar{n}(t)),
$$

where $F(\bar{n}(t))$ is given by (DRIFT). In order to know the average number of stations at each backoff stage at steady state, we can compute the equilibrium point(s) of this system, which is the stationary regime where the average number of stations at each backoff stage remains constant. This information will later enable us to compute actual throughput figures.

Next, we compute the equilibrium point of (6) by imposing $F(\bar{n}(t))=0$, which yields

$$
\begin{aligned}
\bar{n}_{i} & =\left(\frac{\tau_{i-1} p_{i-1}+\beta_{i-1}}{\tau_{i}+\beta_{i}}\right) \bar{n}_{i-1}, \quad 1 \leq i \leq m-2, \\
\bar{n}_{m-1} & =\left(\frac{\tau_{m-2} p_{m-2}+\beta_{m-2}}{\tau_{m-1}\left(1-p_{m-1}\right)}\right) \bar{n}_{m-2} .
\end{aligned}
$$

Let us define

$$
\begin{aligned}
& K_{0}:=1, K_{i}:=\frac{\tau_{i-1} p_{i-1}+\beta_{i-1}}{\tau_{i}+\beta_{i}}, \quad 1 \leq i \leq m-2, \\
& K_{m-1}:=\frac{\tau_{m-2} p_{m-2}+\beta_{m-2}}{\tau_{m-1}\left(1-p_{m-1}\right)} .
\end{aligned}
$$

Since $\sum_{i=0}^{m-1} \bar{n}_{i}=N$, it follows that the equilibrium of system (6) is given by the following system of equations:

$$
\bar{n}_{0}=\frac{N}{\sum_{k=0}^{m-1} \prod_{j=0}^{k} K_{j}}, \bar{n}_{i}=\frac{N \prod_{j=0}^{i} K_{j}}{\sum_{k=0}^{m-1} \prod_{j=0}^{k} K_{j}} 1 \leq i \leq m-1 .
$$

Recall that $\tau_{i}$ and $\beta_{i}$ are functions of $p_{i}$, given by (4) and (5). Thus, the $\bar{n}_{i}$ 's in (EQ) are also functions of $p_{i}, 0 \leq i \leq m-1$. From the above, substituting (EQ) in (1) yields a system of $m$ equations and $m$ unknowns $p_{i}$ for $0 \leq i \leq m-1$. The 
following theorem is one of our main results. It states that, for configurations satisfying $C W_{i+1} \geq 2 C W_{i}-d_{i}-1,0 \leq$ $i<m-1$, the system (EQ), (1) admits exactly one solution, hence that the equilibrium point of (6) is unique. Note that, from Table I, these constraints are compliant with the standard, except for the class CA2/CA3 at backoff stage $i=1$. We leave the extension of the uniqueness result to this priority class, and the investigation of the stability of (6) for future work.

Theorem 1. Assume that $C W_{i} \geq 6$ and $d_{i} \leq\left\lfloor C W_{i} / 2-\right.$ $1\rfloor, 0 \leq i \leq m-1$. In addition, assume that $C W_{i+1} \geq$ $2 C W_{i}-d_{i}-1,0 \leq i<m-1$. Then the system of equations formed by (EQ) and (1) has a unique solution.

Proof: Recall that $p_{e}=\prod_{k=0}^{m-1}\left(1-\tau_{k}\right)^{\bar{n}_{k}}$. For any value of $p_{e}, \tau_{i}$ can be computed from the fixed-point equation that results from combining (1) (i.e., $p_{i}=1-p_{e} /\left(1-\tau_{i}\right)$ ) with (4), where (4) is expressed as a function of $p_{i}$ through (2) (see [16] for more details). Hence, $\tau_{i}$ can be computed as a function of $p_{e}$, and so can $p_{i}, \beta_{i}$. Now, $\bar{n}_{i}$ can also be computed as a function of $p_{e}$, given (EQ). With this, let $\Phi\left(p_{e}\right):=\prod_{k=0}^{m-1}\left(1-\tau_{k}\left(p_{e}\right)\right)^{\bar{n}_{k}\left(p_{e}\right)}$. Then, a solution of (EQ) has to satisfy the following equation:

$$
p_{e}=\Phi\left(p_{e}\right) .
$$

We next show that there exists only one value of $p_{e}$ that satisfies (8). Due to space constraints, we show in [16] that $\Phi\left(p_{e}\right)$ has at least one fixed-point. To this end, we show that $\Phi\left(p_{e}\right)$ is monotonically decreasing with $p_{e}$. The derivative of $\Phi\left(p_{e}\right)$ can be written as

$$
\frac{d \Phi\left(p_{e}\right)}{d p_{e}}=\sum_{j=0}^{m-1}\left(\frac{\partial \Phi}{\partial p_{j}} \frac{\partial p_{j}}{\partial p_{e}}+\frac{\partial \Phi}{\partial \beta_{j}} \frac{\partial \beta_{j}}{\partial p_{e}}+\frac{\partial \Phi}{\partial \tau_{j}} \frac{\partial \tau_{j}}{\partial p_{e}}\right) .
$$

We now examine separately each of the partial derivative products of (9) with respect to $p_{j}, \beta_{j}$ and $\tau_{j}$.

First, Lemmas 1 and 2 in Appendix imply respectively that $\partial p_{j} / \partial \tau_{j}<0$ and $\partial \tau_{j} / \partial p_{e}<0$. Because $\partial p_{j} / \partial p_{e}=$ $\left(\partial p_{j} / \partial \tau_{j}\right) \cdot\left(\partial \tau_{j} / \partial p_{e}\right)$, we have $\partial p_{j} / \partial p_{e}<0$. Also, by Lemma 5 in Appendix, $\partial \Phi / \partial p_{j}>0$. Thus, the first product of partial derivatives in (9) is negative for all $j$. Second, by Lemma 3 in Appendix, $\partial \Phi / \partial \beta_{j} \geq 0$. Now, Corollary 2 states that $\partial \beta_{j} / \partial p_{j}>0$ and we have shown above that $\partial p_{j} / \partial p_{e}<0$. Hence, we have $\partial \beta_{j} / \partial p_{e}<0$, and the second product of partial derivatives in (9) is also negative. Third, by Lemma 4 we have $\partial \Phi / \partial \tau_{j}<0$, and by Lemma 2 we have $\partial \tau_{j} / \partial p_{e}>0$. We thus have shown that all the partial derivative products of (9) are negative, and so $\Phi\left(p_{e}\right)$ is monotonically decreasing with $p_{e}$.

Since (9) is strictly negative, there exists a unique value for $p_{e}$ that solves (8). Computing the corresponding value for $p_{i}$ by (1), we have a solution to (EQ). The uniqueness of the solution then follows from the fact that all relationships between $\tau_{i}, \beta_{i}, p_{i}$ and $p_{e}$ are bijective, and any solution must satisfy (8), which (as we have shown) has only one solution.

In Section V, we observe that our model is very accurate for all configurations. We explain how collision probability and throughput are computed in the same section.

\section{Performance Evaluation}

In this section, we evaluate the 1901 performance under different configurations. First, we validate experimentally our simulator and our model by using a testbed. Second, we evaluate our model and compare it with the models based on the decoupling assumption [6], [7]. These models perform different computations, but are strictly equivalent in terms of predicted throughput. We thus refer to these models as (the unique) "D.A." model.

\section{A. Experimental Validation}

We use simulations to evaluate 1901 performance. We wrote a Matlab simulator, which implements the full CSMA/CA mechanism of $1901^{7}$. In this subsection, we validate the accuracy of our model and simulator with experimental results from a HomePlug AV test-bed.

We built a test-bed of 7 stations, each comprising a PLC interface ${ }^{8}$. The stations are ALIX boards running the OpenWrt Linux distribution [18]. Each board is equipped with a Homeplug AV miniPCI card (Intellon INT6300 chip). In our tests, $N$ stations send UDP traffic (at a rate higher than the link capacities) to the same non-transmitting station using iperf. We run tests for $1 \leq N \leq 6$. At the end of each test we request the number of collided and successfully transmitted frames from each station using the Qualcomm Atheros Open Powerline Toolkit [19]. Using this information, we evaluate the collision probability.

We compare the collision probability measured on the testbed with the one obtained with our model. To this end, we use our model to compute the steady-state expected number of nodes $\bar{n}_{0}, \ldots, \bar{n}_{m-1}$ at each backoff stage. Once we have the average number of stations at each each backoff stage, the probability $p_{i}$ of collision at backoff stage $i$ is readily given by (1).

Let $\gamma$ be the average probability that a transmission in the system collides. The probability that a given transmission in the system corresponds to a station at backoff stage $i$ is given by $\bar{n}_{i} \tau_{i} / \sum_{k=0}^{m-1} \bar{n}_{k} \tau_{k}$. We thus have $\gamma=$ $\sum_{i=0}^{m-1} \bar{n}_{i} \tau_{i} p_{i} / \sum_{i=0}^{m-1} \bar{n}_{i} \tau_{i}$.

The average collision probabilities obtained from 10 testbed experiments, 10 simulation runs, and our model are shown in Figure 4. We observe an excellent fit between experimental and simulation/analytic results.

Contrary to some existing 802.11 interfaces, the MAC parameters of the HomePlug AV devices cannot be modified, because they are stored in the firmware, and the required offsets of their binary values are not publicly available. Therefore, the results on throughput and fairness have been obtained with our validated simulator.

\footnotetext{
${ }^{7}$ Our simulator and the guidelines to reproduce all the testbed experiments of this work are available in [17].

${ }^{8}$ The stations also have a wireless interface, a miniPCI card Atheros DNMA92. This interface is used only for the experiments of Section I. To avoid interference with other devices in our building we set the mode to 802.11a and the wireless channel to 44. To obtain the packet trace of Figures 1, 2 for 802.11a we use tcpdump. To capture the transmitted frames for 1901, we use the tools described in [17], because this MAC employs frame aggregation and the number of Ethernet packets per PLC frame varies with time.
} 


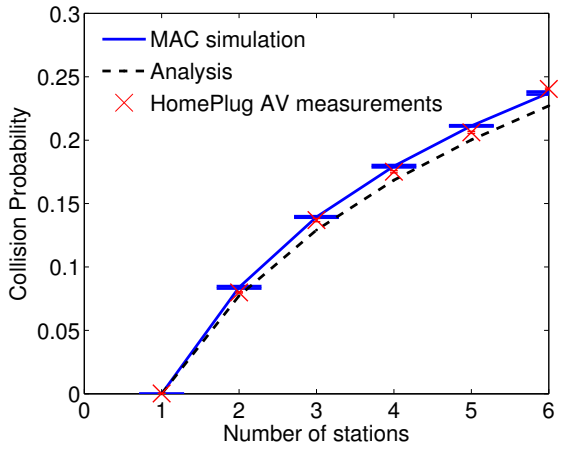

Fig. 4. Collision probability obtained by simulation, our drift model of Section IV and experiments with HomePlug AV devices for the default configuration CA1 of 1901 given in Table I.

\section{B. Simulation Parameters and Throughput Computation}

Our simulator uses the same time slot duration and timing parameters as specified in the standard (see Table III). The PLC frame transmission has a duration $D$ and is preceded by two priority tone slots $(P R S)$, and a preamble $(P)$. It is followed by a response inter-frame space (RIFS), the ACK, and finally, the contention inter-frame space $(C I F S)$. Thus, a successful transmission has a duration $T_{s}:=2 P R S+P+D+R I F S+$ $A C K+C I F S$. In the case of a collision, the stations set the virtual carrier sense (VCS) timer equal to EIFS, where $E I F S$ is the extended inter-frame space used by 1901, and then the channel state is idle. Hence, a collision has a duration $T_{c}:=E I F S$. Finally, we assume that all the packets use the same physical rate.

\begin{tabular}{ll} 
Parameter & Duration $(\mu s)$ \\
\hline \hline Slot $\sigma$, Priority slot PRS & 35.84 \\
$C I F S, R I F S$ & 100.00 \\
Preamble $P, A C K$ & 110.48 \\
Frame duration $D$ & 2050.00 \\
$E I F S$ & 2920.64 \\
\hline
\end{tabular}

TABLE III

SIMULATION PARAMETERS.

To analytically evaluate throughput, we employ the model of Section IV. After solving the equations for finding the steadystate number of nodes $\bar{n}_{0}, \ldots, \bar{n}_{m-1}$ at each backoff stage, we can compute the throughput of the network as follows. The probability that a slot is idle is $p_{e}$. The probability of a successful transmission of a station at stage $i$ is $\tau_{i}\left(1-p_{i}\right)$. Therefore, the probability $p_{s}$ that a slot contains a successful transmission is given by $p_{s}=\sum_{i=0}^{m-1} \bar{n}_{i} \tau_{i}\left(1-p_{i}\right)$. Let $p_{c}$ denote the probability that a slot contains a collision. We have $p_{c}=1-p_{e}-p_{s}$. We now have enough information to compute the normalized throughput $S$ of the network as

$$
S=\frac{p_{s} D}{p_{s} T_{s}+p_{c} T_{c}+p_{e} \sigma},
$$

where $D$ is the frame duration, $T_{s}$ is the duration of a successful transmission, $T_{c}$ is the duration of a collision, and $\sigma$ is the time slot duration.

\section{The Decoupling Assumption Does Not Hold for 1901}

For 802.11, the decoupling assumption has been shown to be viable in various settings as $N \rightarrow \infty$ [11]. In addition,
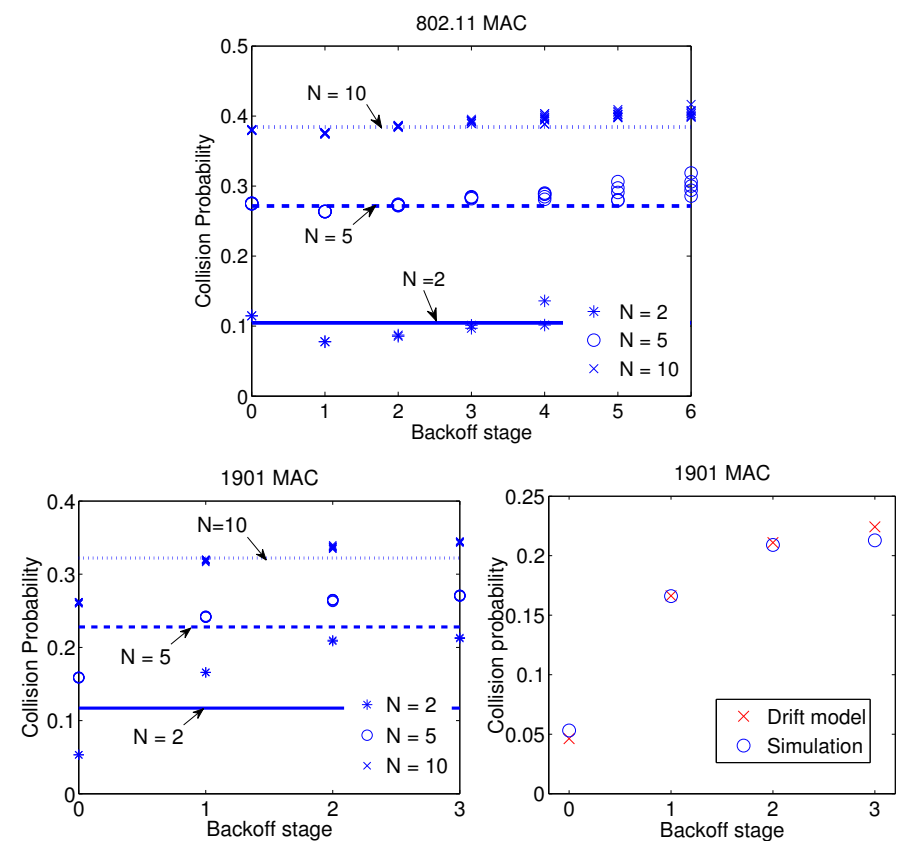

Fig. 5. Simulations of 1901 (with CA1 parameters) and 802.11 for $N=$ $2,5,10$. Points show the collision probabilities at different backoff stages for all stations, and lines represent the solution of the fixed-point equations for the collision probability from the D.A. models [5], [7]. The decoupling assumption is viable for 802.11 even for $N=2$, whereas the collision probability depends on the backoff stage for 1901. Our model accurately predicts the collision probability at each backoff stage $i$ (shown at the right for $N=2$ ).

it turns out that it also works well for small numbers of stations [5], [12]. For 1901, the coupling induced by the deferral counter makes the collision probabilities state-dependent, which penalizes models based on the decoupling assumption when $N$ is small. To see this, we plot on Figure 5 the collision probabilities experienced by 802.11 and 1901 stations, as a function of the backoff stage (i.e., as a function of the stations' state). On the same figure, we also show the collision probabilities computed with our model. Let $C_{k}$ be the sequence of outcomes of attempted transmissions, i.e. $C_{k}:=0$ if the $k$ th transmission attempt results in a success, and $C_{k}:=1$ when the outcome is a collision. The decoupling assumption asserts that the sequence $\left\{C_{k}\right\}$ consists of independent and identically distributed (i.i.d.) random variables. In Figure 5, we observe that for 1901, $\left\{C_{k}\right\}$ cannot be considered as i.i.d., because the collision probability observed at different backoff stages is not the same. The collision probability depends on the previous transmission attempts (backoff stage changed due to collision) or on other stations activity (backoff stage changed due to sensing the medium busy). In fact, the collision probability for 1901 is an increasing sequence of the backoff stage $i$, as shown in Figure 5, and proved in Corollary 1 in Appendix.

\section{Model Evaluation}

We now compare our drift model with the D.A. model for various configurations and number of stations. In Figure 6, we show the throughput obtained by 1901 with the default parameters for the two priority classes CA1 and CA3 (CA0 and CA2 are equivalent). We also show the throughput predicted by the two models. The model based on the decoupling assumption is less accurate for CA1 when $N$ is small, because the class 
CA1 uses larger contention windows, which increases the time spent in backoff and, as a result, the coupling between stations.

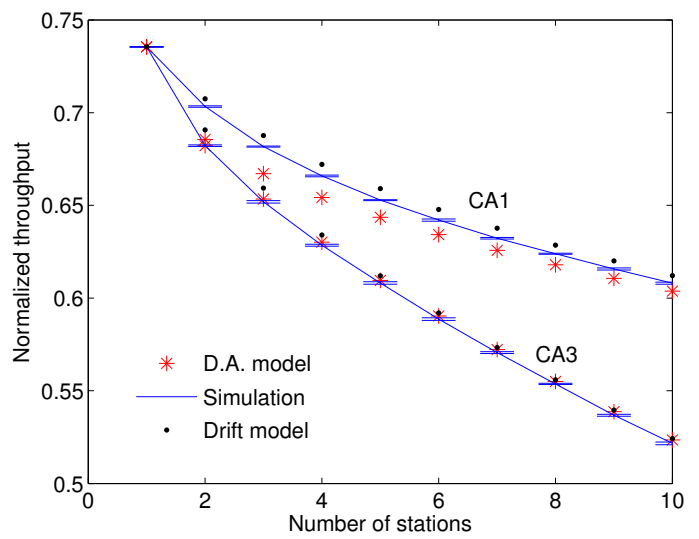

Fig. 6. Throughput obtained by simulation, with our model, and the models based on the decoupling assumption (D.A.), for the default configurations of 1901 given in Table I.

We now study the accuracy of the two models in more general settings. To this end, we introduce a factor $f$, such that at each stage $i$, the value of $d_{i}$ is given by $d_{i}=f^{i}\left(d_{0}+1\right)-1$. This enables us to define various sequences of values for the $d_{i}$ 's, using only $f$ and $d_{0}$. At each stage $i, C W_{i}$ is given by $C W_{i}=2^{i} C W_{\min }$, and there are $m$ backoff stages $(i \in\{0, m-$ $1\})$. In Figure 7, we show the throughput for various such values of $d_{0}$ and $f$, with $C W_{\min }=8$ and $m=4$. We observe that the D.A. model achieves good accuracy when the $d_{i}$ 's are large, because in these configurations, the deferral counter is less likely to expire, which reduces the coupling among stations. Note that the drift model achieves good accuracy when the $d_{i}$ 's are small.

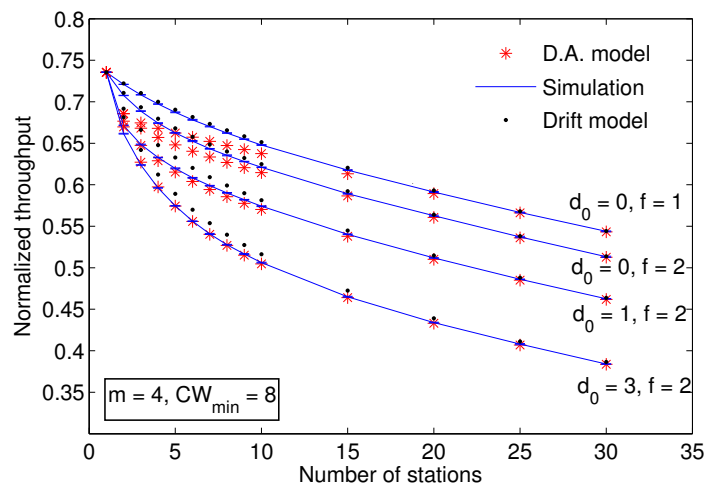

Fig. 7. Throughput obtained by simulation, with the drift model, and the D.A model for different configurations. The initial values $d_{i}$ of the deferral counter at each backoff stage are given by $d_{i}=f^{i}\left(d_{0}+1\right)-1$.

Finally, in Figure 8 we show the throughput for different values for $C W_{\min }$, with $m \in\{4,6\}$. In all cases, the drift model fits very well, contrary to the model based on the decoupling assumption. The accuracy of the D.A. model is penalized more when $C W_{\min }$ is large, because the likelihood that the deferral counter expires increases.

\section{Throughrut, FAIRness AND COUPLing}

In this section we show how the coupling between 1901 stations is related to throughput and jitter (or, equivalently,

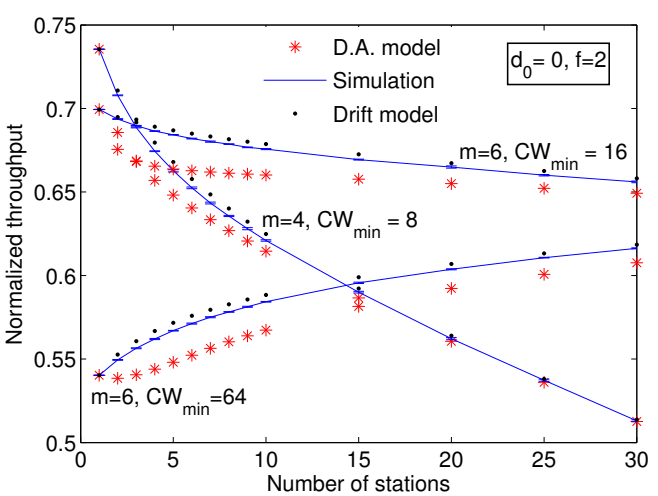

Fig. 8. Throughput obtained by simulation, with the drift model, and the D.A. model for various values of $C W_{\min }$ and $m \in\{4,6\}$.

unfairness). Moreover, based on our model, we propose a method to optimize jitter under a given throughput requirement.

In the introductory example of Figure 1, we observe a bistability effect with two 1901 stations, where stations are likely to remain for long durations in states with large transmit (resp. backoff) probabilities. We explain that this effect is caused by the deferral counter, which creates a coupling between the stations and penalizes the accuracy of models assuming decoupling. It turns out that this coupling is beneficial for throughput ${ }^{9}$, and different 1901 configurations determine different tradeoffs between fairness (or jitter) and throughput. We now investigate this effect further in terms of shortterm fairness. A MAC protocol is short-term fair when the stations get similar transmission opportunities over short time scales. Conversely, an unfair protocol advantages some stations over others, which in practice results in high delay variance (jitter). To measure short-term fairness, we compute Jain's fairness index [20] over windows of $N$ frame durations. More precisely, if we let $x_{i}(w)$ be the number of frames successfully transmitted by station $i$ during a window of $w$ frame durations, Jain's index during window $w$ is defined as $J(w)=\left(\sum_{i=1}^{N} x_{i}(w)\right)^{2} /\left(N \sum_{i=1}^{N} x_{i}(w)^{2}\right)$. In the following, we take $w=N$, as this is the smallest value of $w$ such that $J(w)$ can be equal to 1 (for a perfectly fair protocol). The reported results are obtained by averaging the values of $J(N)$ over windows moving along the whole packet traces.

In Figure 9, we plot throughput and short-term fairness as a function of the initial values of the deferral counter (in terms of $d_{0}$ and $f$ ), for $N=2$ and $N=5$. Interestingly, it appears that that there is a direct link between throughput, fairness and coupling. When the initial deferral counters are small (corresponding to small $d_{0}$ and $f$ ), the stations are more likely to react on sensing the medium busy and thus become coupled. Indeed, as confirmed by Figure 7, the configurations employing small deferral counters yield larger throughput but lower accuracy when assuming decoupling. Conversely, these configurations have the worse short-term fairness and thus cause higher jitter.

We study further the throughput/fairness tradeoff on Fig-

\footnotetext{
${ }^{9}$ Intuitively, this is easy to understand: without proper synchronization, having one station transmitting for long durations is more efficient than alternating transmissions.
} 

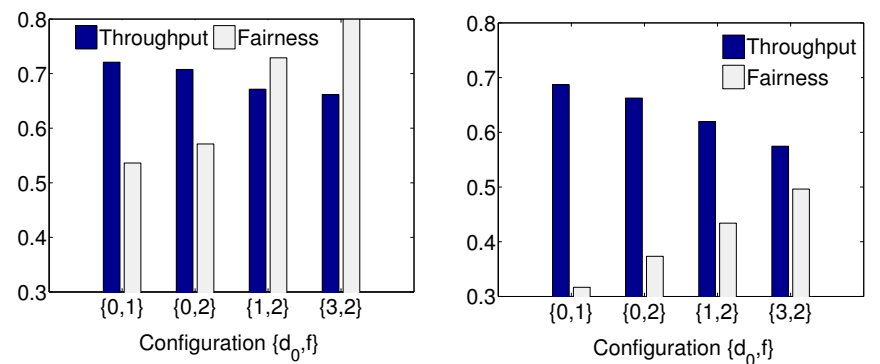

Fig. 9. Throughput and fairness with $C W_{\min }=8$ and $m=4$ and various values of $d_{0}$ and $f$, for $N=2$ (left) and $N=5$ (right). The initial value of the deferral counter at backoff stage $i$ is given by $d_{i}=f^{i}\left(d_{0}+1\right)-1$.
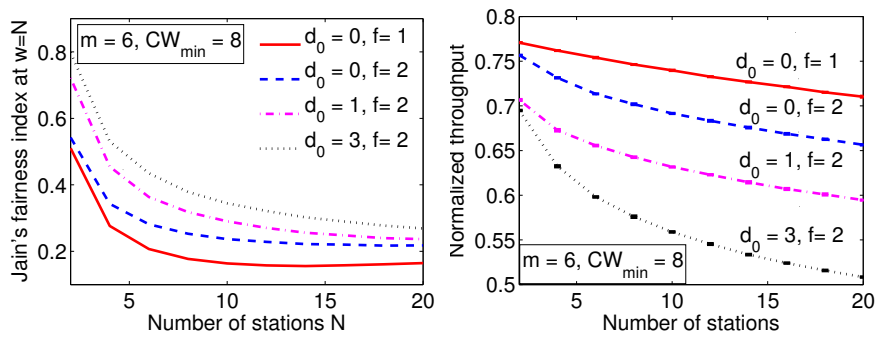

Fig. 10. Short-term fairness and throughput obtained by simulation for parameters $C W_{\min }=8, m=6$ and various values of $d_{0}$ and $f$. The deferral counter tunes a tradeoff between throughput and fairness in 1901 .

ures 10 and 11. Both figures show the throughput and fairness achieved on networks with a varying number of stations. Figure 10 presents throughput and fairness for various initial deferral counter values (in terms of $d_{0}$ and $f$ ). Figure 11 shows these two metrics for different numbers of backoff stages $m$.

Again, both figures show a clear tradeoff between throughput and short-term fairness. Furthermore, this tradeoff can be tuned by adapting the parameters that control the number of backoff stages and the initial values of the deferral counters. This possibility is a remarkable feature of 1901, enabled by the deferral counter. We summarize the impact of all parameters on throughput and fairness in Table IV.

\section{A. Finding Efficient Configurations}

We can use the findings summarized in Table IV, together with our drift model, to find efficient configurations that meet specific QoS criteria. For example, we propose a simple heuristic algorithm that finds an efficient configuration in terms of jitter, given an arbitrary throughput requirement (if such a configuration exists). Our method is detailed in Algorithm 1 and works as follows. It orders (by increasing order of values) the sets of possible values taken by $d_{0}$ and $m$ in two sequences named $\mathcal{D}$ and $\mathcal{M}$, respectively. It then performs a binary search on $\mathcal{D}$ : for a given $d_{0}$ in $\mathcal{D}$, it tests all combination of parameters $\left(m, C W_{\min }\right)$ (by increasing order of $m$ ). When such a configuration satisfies the throughput requirement, the algorithm stores it and tries a larger value for $d_{0}$ (as a larger $d_{0}$ can potentially yield better jitter). Conversely, if no configuration meeting the throughput requirement is found, the algorithm considers smaller values for $d_{0}$ (which yield higher throughputs, potentially at the expense of jitter). The algorithm ends when it finds the best configuration that corresponds to the largest possible $d_{0}$ that satisfies the constraint.
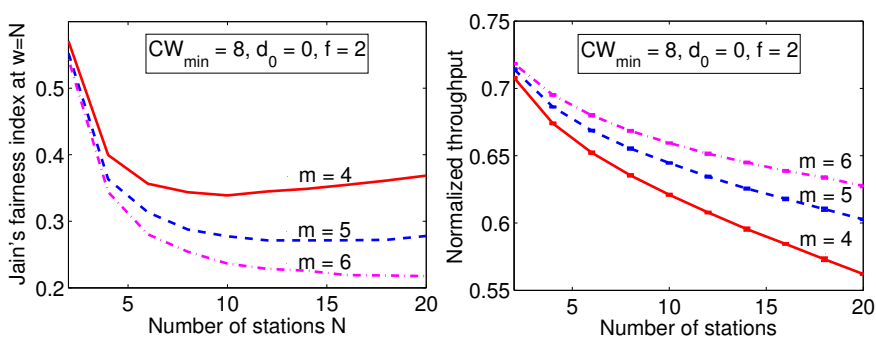

Fig. 11. Short-term fairness and throughput obtained by simulation with parameters $C W_{\min }=8, d_{0}=0, f=2$ and various values of $m$.

\begin{tabular}{l||l|l|l|l} 
& $d_{0}$ & $f$ & $m$ & $C W_{\min }$ \\
\hline \multirow{2}{*}{ small } & $\mathrm{T} \nearrow$ & $\mathrm{T} \nearrow$ & $\mathrm{T} \searrow$ & $\mathrm{T} \nearrow$ if $N$ is small \\
& $\mathrm{F} \searrow$ & $\mathrm{F} \searrow$ & $\mathrm{F} \nearrow$ & $\mathrm{F} \rightarrow$ \\
\hline \multirow{2}{*}{ large } & $\mathrm{T} \searrow$ & $\mathrm{T} \searrow$ & $\mathrm{T} \nearrow$ & $\mathrm{T} \nearrow$ if $N$ is large \\
& $\mathrm{F} \nearrow$ & $\mathrm{F} \nearrow$ & $\mathrm{F} \searrow$ & $\mathrm{F} \rightarrow$
\end{tabular}

TABLE IV

SUMMARY OF THE QUALITATIVE EFFECTS OF EACH PARAMETER ON THROUGHPUT ("T") AND SHORT-TERM FAIRNESS ("F").

Because it employs a binary search, the complexity of this algorithm is $O(|\mathcal{C}| \cdot|\mathcal{M}| \cdot \log (|\mathcal{D}|))$. We evaluate it on the sequences $\mathcal{C}=(8,16,32,64), \mathcal{M}=(4,5,6)$ and $\mathcal{D}=(0,1,2,3)^{10}$. First, we run simulations of all the possible configurations in $\{\mathcal{C} \times \mathcal{M} \times \mathcal{D}\}$, and we compute short-term fairness $J(N)$ and normalized throughput $S$. Let $S_{\text {min }}$ be the minimum $S$ achieved by all configurations in $\{\mathcal{C} \times \mathcal{M} \times \mathcal{D}\}$, and similarly $S_{\max }$ be the maximum $S$. To test our algorithm, we draw 100 throughput requirements uniformly at random in $\left[S_{\min }, S_{\max }\right]$. Then, for each sample $i$ with throughput $S_{i}$, we run Algorithm 1 that returns the configuration $\operatorname{config}_{i}$. Now, let $J_{i}$ be the short-term fairness of the configuration con $i g_{i}$ at sample $i$, and let $J_{i}^{\max }$ denote the maximum short-term fairness of all configurations that satisfy the throughput constraint $S_{i}$. To evaluate the algorithm, we employ a normalized fairness index that is defined as $J_{i} / J_{i}^{\max }$. The normalized fairness is a metric that evaluates the distance between the fairness of the configuration con $\mathrm{fig}_{i}$ and the maximum achievable fairness given the the throughput constraint $S_{i}$.

The results of the algorithm evaluation are presented in Figure 12. We present the normalized fairness of the configurations returned from 100 runs of Algorithm 1. We repeat the procedure described above for $2 \leq N \leq 8$. We observe that Algorithm 1 always returns a configuration with good fairness given the throughput constraint. Thus, it can be employed to optimize the performance for delay-sensitive traffic that operates with 1901.

\section{CONCLUSION}

The IEEE 1901 CSMA/CA protocol has received little attention from the research community so far, although it is adopted by the vast majority of power-line communication devices. In this paper, we focus on the analysis of the performance of this protocol. One of the key results is the

\footnotetext{
${ }^{10}$ We use the factor $f=2$ because the contention windows are also doubled between successive backoff stages. Algorithm 1 can be modified to include different $f$ values given the performance tradeoff of $f$ in Table IV.
} 

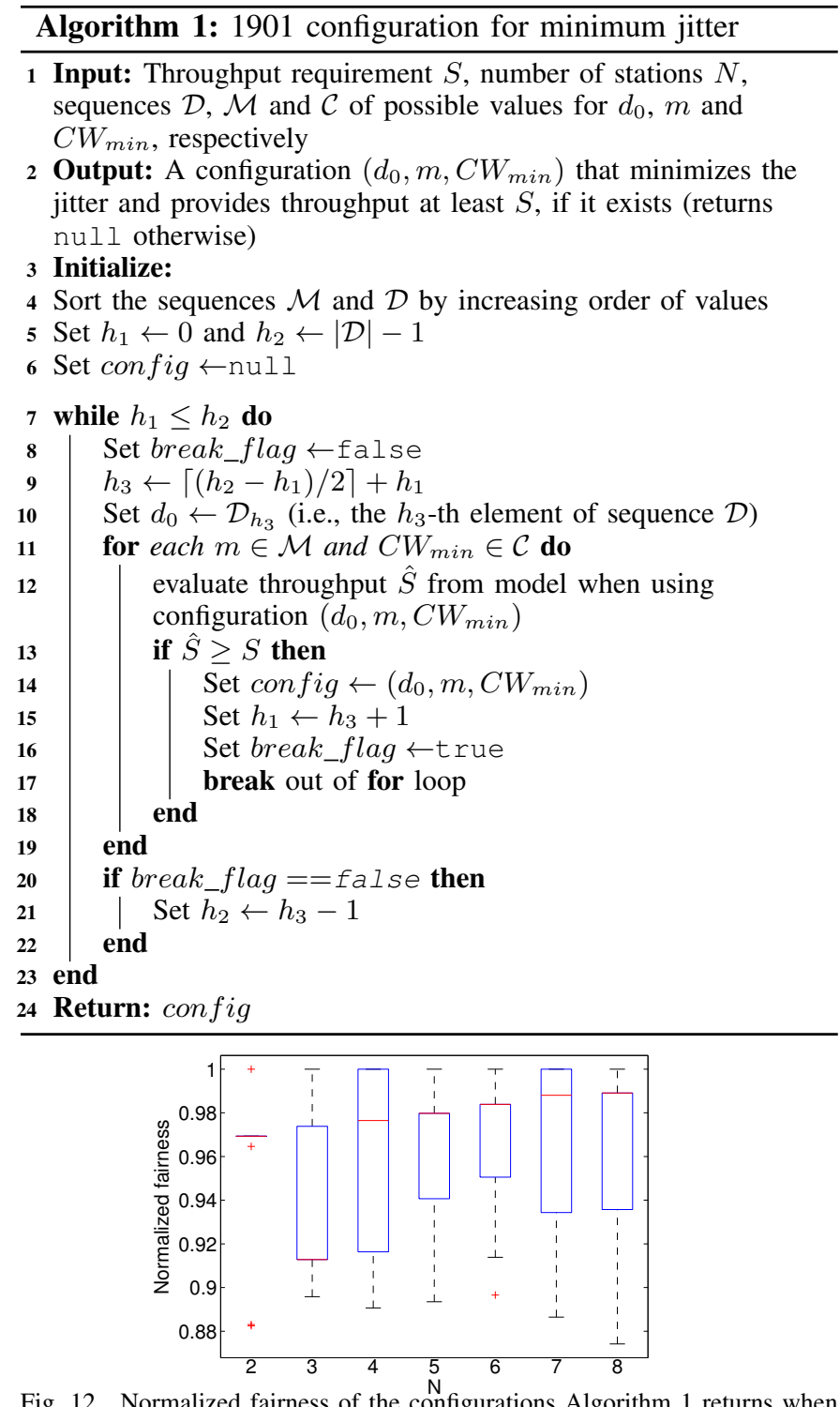

Fig. 12. Normalized fairness of the configurations Algorithm 1 returns when run for 100 throughput requirements $S$ chosen randomly, for each $N$ value.

finding that the decoupling assumption, which is commonly adopted for the analysis of MAC protocols such as IEEE 802.11 and IEEE 1901, might not hold for 1901. This is due to the coupling that this protocol introduces to the stations contending for the medium. Building on this finding, we have proposed a new model that does not rely on the decoupling assumption and thus substantially improves the accuracy of previous studies, in particular for networks with a small number of stations, the most frequent configuration in practice. Our model reveals that, as a result of the coupling between stations, 1901 suffers from short-term unfairness. To address this issue, we have explored the tradeoff between short-term fairness and throughput that exists in 1901. In this context, we devised a method that computes efficient configurations in terms of fairness/jitter, under arbitrary throughput constraints.

\section{APPENDIX}

We provide the sketch of the proof of some necessary lemmas for Theorem 1. The detailed proofs can be found in [16].
Lemma 1. $\tau_{i}$ is a decreasing function of $p_{i}$ for any $i$.

Proof: The probability $\tau_{i}$ given by (4) can be recast as

$$
\tau_{i}=\frac{1}{B_{i}+1}
$$

where $B_{i}$ is the expected number of backoff slots between two transmission attempts of a station that always stays at backoff stage $i . B_{i}$ can be computed recursively and is given by

$$
\begin{aligned}
B_{i} & =\frac{d_{i}\left(d_{i}+1\right)}{2 C W_{i}} \\
+ & \sum_{j=d_{i}+1}^{C W_{i}-1} \frac{j\left(1-x_{j}^{i}\right)+\sum_{k=d_{i}+1}^{j}\left(k+B_{i}\right)\left(x_{k}^{i}-x_{k-1}^{i}\right)}{C W_{i}} .
\end{aligned}
$$

To prove the lemma, we need to show that $B_{i}$ is an increasing function of $p_{i}$. To do this, we proceed as follows. (i) First, we compute the derivative of $B_{i}$ with respect to $p_{i}$. (ii) Second, we show that this derivative is positive at $p_{i}=1$. (iii) Third, we further show that if the derivative is negative for some $0<p_{i}^{*}<1$, it will also be negative at any value $p_{i}>p_{i}^{*}$. The proof then follows by contradiction: If the derivative was negative at some $p_{i}^{*}$, it would also be negative at $p_{i}=1$, which would contradict our previous result (ii).

(i) After simple manipulations, (12) can be recast as

$$
B_{i}=\frac{C W_{i}-1}{2}+\frac{1}{C W_{i}} \sum_{j=d_{i}+1}^{C W_{i}-1}\left(B_{i} x_{j}^{i}-\sum_{k=d_{i}+1}^{j-1} x_{k}^{i}\right) .
$$

The derivative of $B_{i}$ can be computed as

$$
\frac{d B_{i}}{d p_{i}}=\sum_{k=d_{i}+1}^{C W_{i}-1} \frac{\partial B_{i}}{\partial x_{k}^{i}} \frac{\partial x_{k}^{i}}{\partial p_{i}} .
$$

The partial derivative $\partial B_{i} / \partial x_{k}^{i}$ can be computed from (13) as

$$
\frac{\partial B_{i}}{\partial x_{k}^{i}}=\frac{B_{i}-\left(C W_{i}-1-k\right)}{C W_{i}}+\frac{\partial B_{i}}{\partial x_{k}^{i}} \sum_{j=d_{i}+1}^{C W_{i}-1} \frac{x_{j}^{i}}{C W_{i}},
$$

which yields

$$
\frac{\partial B_{i}}{\partial p_{i}}=\frac{\sum_{k=d_{i}+1}^{C W_{i}-1}\left(B_{i}-\left(C W_{i}-1-k\right)\right) \frac{\partial x_{k}^{i}}{\partial p_{i}}}{C W_{i}-\sum_{j=d_{i}+1}^{C W_{i}-1} x_{j}^{i}} .
$$

To compute $\partial x_{k}^{i} / \partial p_{i}$, we observe that $x_{k}^{i}$ is the complementary cumulative function of a binomial distribution. By taking its partial derivative, we obtain

$$
\frac{\partial x_{k}^{i}}{\partial p_{i}}=\frac{k !}{\left(k-d_{i}-1\right) ! d_{i} !} p_{i}^{d_{i}}\left(1-p_{i}\right)^{k-d_{i}-1},
$$

which ends the computation of $d B_{i} / d p_{i}$.

(ii) Next, we show that $d B_{i} / d p_{i}>0$ at $p_{i}=1$. We have

$$
B_{i}=\frac{d_{i}\left(d_{i}+1\right)}{2 C W_{i}}+\frac{C W_{i}-d_{i}-1}{C W_{i}}\left(d_{i}+1+B_{i}\right) .
$$

Solving (18) over $B_{i}$ yields $B_{i}=C W_{i}-d_{i} / 2-1$ at $p_{i}=1$. Substituting in (16), it follows that $d B_{i} / d p_{i}=d_{i} / 2+1>0$ at $p_{i}=1$, which completes step (ii). 
(iii) Finally, we show that if $d B_{i} / d p_{i}$ was negative at some value $p_{i}^{*}$, then it would also be negative for any larger value of $p_{i}$. Observe that in (16) some terms are negative for $k<C W_{i}-$ $1-B_{i}$. Let us assume that the derivative is negative at $p_{i}^{*}$. Then, at some $p_{i}=p_{i}^{*}+\epsilon, \epsilon>0$, we have $B_{i}\left(p_{i}\right)<B_{i}\left(p_{i}^{*}\right)$. This implies that in (16) some of the terms that were positive with $p_{i}=p_{i}^{*}$ become negative for $p_{i}>p_{i}^{*}$. Furthermore, from (17) we have that

$$
\frac{\partial x_{k}^{i} / \partial p_{i}}{\partial x_{k-1}^{i} / \partial p_{i}}=\frac{k}{k-d_{i}-1}\left(1-p_{i}\right)
$$

which implies that in (16) the relative weight of the negative terms increases and that of the positive terms decreases.

From the above, it follows that if $d B_{i} / d p_{i}$ was negative at any $p_{i}^{*}$, it would also be negative for all $p_{i}>p_{i}^{*}$. Since this contradicts the above result that $d B_{i} / d p_{i}$ is positive at $p_{i}=1$, we conclude that $d B_{i} / d p_{i} \geq 0$ for $p_{i} \in[0,1]$.

Corollary 1. $\tau_{i}$ is a strictly decreasing sequence of $i$, and $p_{i}$ is a strictly increasing sequence of $i$ if $C W_{i+1} \geq 2 C W_{i}-$ $d_{i}-1,0 \leq i<m-1$.

Proof: The minimum value of $B_{i+1}$ is $B_{i+1}^{\min }:=$ $\left(C W_{i+1}-1\right) / 2$ at $p_{i+1}=0$, and the maximum value of $B_{i}$ is $B_{i}^{\max }:=C W_{i}-d_{i} / 2-1$ at $p_{i}=1$. Setting $C W_{i+1} \geq 2 C W_{i}-d_{i}-1$, yields $B_{i+1}^{\min } \geq B_{i}^{\max }$, hence $B_{i+1}>B_{i}$ and $\tau_{i+1}<\tau_{i}$. Now, $p_{i}$ is strictly increasing with $i$ because $p_{i}=1-p_{e} /\left(1-\tau_{i}\right)$.

Corollary 2. $\beta_{i}$ is an increasing function of $p_{i}$.

Proof: From (4) and (5) we have $\beta_{i}=1 / b c_{i}-\tau_{i}$. (3) can be recast as $b c_{i}=\left(C W_{i}+1\right) / 2-\sum_{k=d_{i}+1}^{C W_{i}-1} \sum_{j=d_{i}+1}^{k} x_{j}^{i} / C W_{i}$. By (17) we have $d x_{k}^{i} / d p_{i}>0$, hence $b c_{i}$ is decreasing with $p_{i}$. Using Lemma $1, \beta_{i}=1 / b c_{i}-\tau_{i}$ is increasing with $p_{i}$.

Lemma 2. Let us consider the expression of $\tau_{i}$ as a function of $p_{e}$ resulting from combining (4) with (1). According to this expression, $\tau_{i}$ is an increasing function of $p_{e}$, if $C W_{i} \geq 6$ and $d_{i} \leq\left\lfloor C W_{i} / 2-1\right\rfloor$.

Proof: The details on how $\tau_{i}$ can be expressed as a function of $p_{e}$ can be found in [16]. Since $\tau_{i}=1 /\left(B_{i}+1\right)$, we need to show that $\partial B_{i} / \partial p_{e}<0$. Note that

$$
\frac{\partial B_{i}}{\partial p_{e}}=\frac{\partial B_{i}}{\partial p_{i}} \frac{\partial p_{i}}{\partial p_{e}}
$$

Because $p_{i}=1-p_{e} /\left(1-\tau_{i}\right)=1-p_{e}\left(B_{i}+1\right) / B_{i}$, we have

$$
\frac{\partial p_{i}}{\partial p_{e}}=-\frac{B_{i}+1}{B_{i}}+\frac{p_{e}}{B_{i}^{2}} \frac{\partial B_{i}}{\partial p_{e}} .
$$

Combining (20) and (21) yields

$$
\frac{\partial B_{i}}{\partial p_{e}}=-\frac{\partial B_{i}}{\partial p_{i}} \frac{B_{i}+1}{B_{i}} \frac{1}{1-\frac{p_{e}}{B_{i}^{2}} \frac{\partial B_{i}}{\partial p_{i}}} .
$$

Because of Lemma $1, \partial B_{i} / \partial p_{i}>0$, and therefore $\partial B_{i} / \partial p_{e}<0$ as long as

$$
\frac{\partial B_{i}}{\partial p_{i}}<\frac{B_{i}^{2}}{p_{e}}=\frac{B_{i}\left(B_{i}+1\right)}{1-p_{i}} .
$$

From (16) we have

$$
\frac{\partial B_{i}}{\partial p_{i}}<\frac{B_{i}}{C W_{i}-\sum_{k=d_{i}+1}^{C W_{i}-1} x_{k}^{i}} \sum_{k=d_{i}+1}^{C W_{i}-1} \frac{\partial x_{k}^{i}}{\partial p_{i}} .
$$

First, let us study (24) with $d_{i}=0$. We have $\partial x_{k}^{i} / \partial p_{i}=$ $k\left(1-p_{i}\right)^{k-1}$ and $x_{k}^{i}=1-\left(1-p_{i}\right)^{k}$. Thus, (24) yields

$$
\frac{\partial B_{i}}{\partial p_{i}}<\frac{B_{i}}{1-p_{i}} \frac{\sum_{k=0}^{C W_{i}-1} k\left(1-p_{i}\right)^{k}}{\sum_{k=0}^{C W_{i}-1}\left(1-p_{i}\right)^{k}} \leq \frac{B_{i}^{2}}{1-p_{i}} .
$$

Define $h\left(p_{i}\right):=\sum_{k=0}^{C W_{i}-1} k\left(1-p_{i}\right)^{k} / \sum_{k=0}^{C W_{i}-1}\left(1-p_{i}\right)^{k}$. The last inequality in (25) holds because first, $h$ is strictly decreasing with $p_{i}$ in $[0,1]$ using Lemma 5.1 in [10], hence $h\left(p_{i}\right) \leq h(0)=\left(C W_{i}-1\right) / 2$. Second, $B_{i}\left(p_{i}\right) \geq\left(C W_{i}-1\right) / 2$ using the proof of Lemma 1 .

We now move to the case $d_{i} \neq 0$. We show that $\partial B_{i} / \partial p_{i}<$ $B_{i}^{2}$, which is sufficient for (23) to hold. From (17) and (2), we have $\partial x_{k}^{i} / \partial p_{i}=k\left(x_{k}^{i}-x_{k-1}^{i}\right) / p_{i}$. Thus, (24) yields

$$
\frac{\partial B_{i}}{\partial p_{i}}<\frac{B_{i}}{p_{i}} \frac{C W_{i} x_{C W_{i}-1}^{i}-\sum_{k=d_{i}+1}^{C W_{i}-1} x_{k}^{i}}{C W_{i}-\sum_{k=d_{i}+1}^{C W_{i}-1} x_{k}^{i}} \leq \frac{B_{i}}{p_{i}} x_{C W_{i}-1}^{i} .
$$

Let $g\left(p_{i}\right)=B_{i} p_{i}-x_{C W_{i}-1}^{i}$. Now, it is sufficient to show that $\partial g / \partial p_{i}>0$ for $p_{i} \in(0,1]$, because $g(0)=0$. Let $X$ be a random variable following the binomial distribution $\operatorname{Bin}\left(C W_{i}-\right.$ $\left.2, p_{i}\right)$. Then, observe that

$$
\frac{\partial g}{\partial p_{i}}=B_{i}+\frac{\partial B_{i}}{\partial p_{i}} p_{i}-\left(C W_{i}-1\right) \mathbb{P}\left(X=d_{i}\right) .
$$

Since $B_{i} \geq\left(C W_{i}-1\right) / 2, \partial g / \partial p_{i}>0$ if $\mathbb{P}\left(X=d_{i}\right)<1 / 2$. The maximum of $\mathbb{P}\left(X=d_{i}\right)$ is at $p_{i}=d_{i} /\left(C W_{i}-2\right)$. We show that $\mathbb{P}\left(Y=d_{i}\right)<1 / 2$, where $Y$ now is a random variable following the distribution $\operatorname{Bin}\left(C W_{i}-2, d_{i} /\left(C W_{i}-2\right)\right)$. Theorem 2.1 in [21] states that, for $n \geq 4$ and $2 \leq k \leq\lfloor n / 2\rfloor$,

$$
\left(\begin{array}{l}
n \\
k
\end{array}\right)<\left(1-\frac{5(k-1)}{6 n^{2}}\right) \frac{n(n-1)^{n-1}}{k^{k}(n-k)^{n-k}} .
$$

With $n=C W_{i}-2$ and $k=d_{i}$, this yields

$$
\mathbb{P}\left(Y=d_{i}\right)<\left(1-\frac{5\left(d_{i}-1\right)}{6\left(C W_{i}-2\right)^{2}}\right)\left(1-\frac{1}{C W_{i}-2}\right)^{C W_{i}-3} .
$$

The above is smaller than 0.42 because $C W_{i} \geq 6$.

Let $\Phi\left(p_{e}\right):=\prod_{k=0}^{m-1}\left(1-\tau_{k}\left(p_{e}\right)\right)^{\bar{n}_{k}\left(p_{e}\right)}$. The following lemmas examine the function $\Phi\left(p_{e}\right)$ where each $\bar{n}_{k}\left(p_{e}\right)$ is a function of all $\beta_{i}\left(p_{e}\right), p_{i}\left(p_{e}\right), \tau_{i}\left(p_{e}\right), 0 \leq i \leq m-1$ by (EQ).

Lemma 3. Let $\Phi\left(p_{e}\right)=\prod_{k=0}^{m-1}\left(1-\tau_{k}\left(p_{e}\right)\right)^{\bar{n}_{k}\left(p_{e}\right)}$, where each $\bar{n}_{k}\left(p_{e}\right)$ is a function of all $\beta_{i}\left(p_{e}\right), p_{i}\left(p_{e}\right), \tau_{i}\left(p_{e}\right), 0 \leq$ $i \leq m-1$. Then, $\partial \Phi / \partial \beta_{j}>0$, for any $0 \leq j<m-1$, and $\partial \Phi / \partial \beta_{m-1}=0$, if $C W_{i+1} \geq 2 C W_{i}-d_{i}-1,0 \leq i<m-1$.

Proof: We consider the expression $\prod_{k=0}^{m-1}\left(1-\tau_{k}\right)^{\bar{n}_{k}}$ as a function of $\tau_{i}, p_{i}$ and $\beta_{i}$, where $\bar{n}_{i}$ is computed as a function of $\tau_{i}, \beta_{i}$ and $p_{i}$ from (EQ). We show that if we increase $\beta_{j}$ for a given $j$, and leave the remaining $\tau_{i}, p_{i}$ and $\beta_{i}$ values fixed, then $\prod_{k=0}^{m-1}\left(1-\tau_{k}\right)^{\bar{n}_{k}}$ increases. From (7), it can be seen that the new $K_{i}$ values, denoted by $K_{i}^{*}$, satisfy the following. 
If $j=0$, then $K_{1}^{*}>K_{1}$ and $K_{i}^{*}=K_{i}, i>1$ by (7). Thus, $\bar{n}_{0}^{*}<\bar{n}_{0}$ and $\bar{n}_{i}^{*}>\bar{n}_{i}, 0<i \leq m-1$. If $j=m-1$, then $K_{i}^{*}=K_{i}$ and $\bar{n}_{i}^{*}=\bar{n}_{i}, 0 \leq i \leq m-1$.

Now, for $1 \leq j \leq m-2$, we have $\prod_{n=1}^{i} K_{n}^{*}=$ $\prod_{n=1}^{i} K_{n}, i<j$ and $\prod_{n=1}^{j} K_{n}^{*}<\prod_{n=1}^{j} K_{n}$.

We also have $\prod_{n=1}^{i} K_{n}^{*}>\prod_{n=1}^{i} K_{n}, i>j$, because

$$
\frac{\prod_{n=1}^{i} K_{n}^{*}}{\prod_{n=1}^{i} K_{n}}=\frac{\frac{\tau_{j} p_{j}+\beta_{j}^{*}}{\tau_{j}+\beta_{j}^{*}}}{\frac{\tau_{j} p_{j}+\beta_{j}}{\tau_{j}+\beta_{j}}}, \frac{\partial}{\partial \beta_{j}}\left(\frac{\tau_{j} p_{j}+\beta_{j}}{\tau_{j}+\beta_{j}}\right)=\frac{\tau_{j}\left(1-p_{j}\right)}{\left(\tau_{j}+\beta_{j}\right)^{2}}>0 .
$$

Let $\sigma:=\sum_{i=1}^{m-1} \prod_{n=1}^{i} K_{n}$. We now show that $\sigma^{*}>\sigma$. We need to show that $\partial \sigma / \partial \beta_{j}>0$. For $j=m-2, \partial \sigma / \partial \beta_{j}>0$ if and only if $\tau_{m-2}\left(1-p_{m-2}\right)-\tau_{m-1}\left(1-p_{m-1}\right)>0$, which holds by Corollary 1 . For $j<m-2$, we prove $\sigma^{*}>\sigma$ by induction. We first show that $\sigma^{*}>\sigma$ for $j=m-3$, and then prove that if it holds for $j=k$, then it holds for $j=k-1$. Taking $\partial \sigma / \partial \beta_{j}$ we find that we need to show that:

$$
\tau_{j}\left(1-p_{j}\right)\left(1+\sum_{i=j+2}^{m-1} \prod_{n=j+2}^{i} K_{n}\right)-\tau_{i+1}-\beta_{i+1}>0 .
$$

For $j=m-3$, (27) holds because of Corollary 1.

Now assume that $\sigma^{*}>\sigma$ for $j=k$. We show that $\sigma^{*}>\sigma$ holds also for $j=k-1$. Let us study (27) for $j=k-1$ :

$$
\begin{aligned}
& \tau_{k-1}\left(1-p_{k-1}\right)\left(1+\sum_{i=k+1}^{m-1} \prod_{n=k+1}^{i} K_{n}\right)-\tau_{k}-\beta_{k} \\
& >\tau_{k-1}\left(1-p_{k-1}\right)\left(1+K_{k+1} \frac{\tau_{k+1}+\beta_{k+1}}{\tau_{k}\left(1-p_{k}\right)}\right)-\tau_{k}-\beta_{k}>0
\end{aligned}
$$

using (27) for $j=k$, (7), and Corollary 1.

Finally, given $\sigma^{*}>\sigma$ we have shown that $\bar{n}_{i}^{*}<\bar{n}_{i}, i \leq j$. Clearly, since $\bar{n}_{i}^{*}<\bar{n}_{i}, i \leq j$ and $\sum_{k} \bar{n}_{k}^{*}=\sum_{k} \bar{n}_{k}=N$, there must be an $l>j$ such that $\bar{n}_{l}^{*}>\bar{n}_{l}$. Since for $i \geq l+1$, $\bar{n}_{i}=K_{i} \bar{n}_{i-1}$ with $K_{i}^{*}=K_{i}$, it holds $\bar{n}_{i}^{*}>\bar{n}_{i}, i>l$. Thus,

$$
\begin{aligned}
& \frac{\prod_{k=0}^{m-1}\left(1-\tau_{k}\right)^{\bar{n}_{k}^{*}}}{\prod_{k=0}^{m-1}\left(1-\tau_{k}\right)^{\bar{n}_{k}}}=\prod_{k<l}\left(1-\tau_{k}\right)^{\bar{n}_{k}^{*}-\bar{n}_{k}} \prod_{k \geq l}\left(1-\tau_{k}\right)^{\bar{n}_{k}^{*}-\bar{n}_{k}} \\
& >\left(1-\tau_{l-1}\right)^{\sum_{k<l} \bar{n}_{k}^{*}-\bar{n}_{k}}\left(1-\tau_{l}\right)^{\sum_{k \geq l} \bar{n}_{k}^{*}-\bar{n}_{k}} .
\end{aligned}
$$

Since $\sum_{k} \bar{n}_{k}^{*}=\sum_{k} \bar{n}_{k}=N$ and $\tau_{l}<\tau_{l-1}$, the above is larger than 1 , which proves the lemma for $0 \leq j \leq m-2$.

Lemma 4. Let $\Phi\left(p_{e}\right)=\prod_{k=0}^{m-1}\left(1-\tau_{k}\left(p_{e}\right)\right)^{\bar{n}_{k}\left(p_{e}\right)}$, defined as in Lemma 3. Then, $\partial \Phi / \partial \tau_{j}<0$, for any $0 \leq j \leq m-1$, if $C W_{i+1} \geq 2 C W_{i}-d_{i}-1,0 \leq i<m-1$.

Proof: When $\tau_{j}$ increases to $\tau_{j}^{*}, \prod_{n=1}^{i} K_{n}^{*}=\prod_{n=1}^{i} K_{n}$ for $i<j$, and $\prod_{n=1}^{i} K_{n}^{*}<\prod_{n=1}^{i} K_{n}$ for $i \geq j$. Hence, it follows that $\prod_{k \neq j}\left(1-\tau_{k}\right)^{\bar{n}_{k}}$ decreases. If we show that $\left(1-\tau_{j}\right)^{\bar{n}_{j}}$ also decreases, the lemma will be proven. Note that

$\frac{\partial\left(1-\tau_{j}\right)^{\bar{n}_{j}}}{\partial \tau_{j}}=-\bar{n}_{j}\left(1-\tau_{j}\right)^{\bar{n}_{j}-1}+\ln \left(1-\tau_{j}\right) \frac{\partial \bar{n}_{j}}{\partial \tau_{j}}\left(1-\tau_{j}\right)^{\bar{n}_{j}}$.

Performing the partial derivative of $\bar{n}_{j}$ we have

$$
\frac{\partial \bar{n}_{j}}{\partial \tau_{j}}=-\frac{\bar{n}_{j}}{\tau_{j}+\beta_{j}}+\frac{\partial \bar{n}_{j-1}}{\partial \tau_{j}} K_{j} \geq-\frac{\bar{n}_{j}}{\tau_{j}+\beta_{j}} \geq-\frac{\bar{n}_{j}}{\tau_{j}} .
$$

Combining the two equations above yields

$$
\frac{\partial\left(1-\tau_{j}\right)^{\bar{n}_{j}}}{\partial \tau_{j}} \leq \frac{\bar{n}_{j}\left(1-\tau_{j}\right)^{\bar{n}_{j}}}{\tau_{j}}\left(-\frac{\tau_{j}}{1-\tau_{j}}-\ln \left(1-\tau_{j}\right)\right) .
$$

Since $-x /(1-x)<\ln (1-x)$, it follows that the above is smaller than 0 , which proves the lemma.

Lemma 5. Let $\Phi\left(p_{e}\right)=\prod_{k=0}^{m-1}\left(1-\tau_{k}\left(p_{e}\right)\right)^{\bar{n}_{k}\left(p_{e}\right)}$, defined as in Lemma 3. Then, $\partial \Phi / \partial p_{j}>0$, for any $0 \leq j \leq m-1$, if $C W_{i+1} \geq 2 C W_{i}-d_{i}-1,0 \leq i<m-1$.

\section{Proof: Similar to Lemma 3.}

\section{ACKNOWLEDGMENT}

This work is financially supported by a grant of the SmartWorld project of the Hasler Foundation, Bern, Switzerland.

\section{REFERENCES}

[1] HomePlug Alliance (retrieved 4/2014). https:/www.homeplug.org/news/ $\mathrm{pr} / \mathrm{view}$ ?item_key=1baefc307e4d175c53568548371cb1142772f4ca.

[2] A. Schwager, "An Overview of the HomePlug AV2 Technology," Journal of Electrical and Computer Engineering, vol. 2013, 2013.

[3] HomePlug Alliance (retrieved 4/2014). http://www.homeplug.org/tech/ whitepapers/Connected_Home_Summits_2013.pdf.

[4] "IEEE Standard for Broadband over Power Line Networks: Medium Access Control and Physical Layer Specifications," IEEE Std 1901-2010.

[5] G. Bianchi, "Performance analysis of the IEEE 802.11 distributed coordination function," Selected Areas in Communications, IEEE Journal on, vol. 18, no. 3, pp. 535-547, 2000.

[6] M. Chung, M. Jung, T. Lee, and Y. Lee, "Performance analysis of HomePlug 1.0 MAC with CSMA/CA," Selected Areas in Communications, IEEE Journal on, vol. 24, no. 7, pp. 1411-1420, 2006.

[7] C. Vlachou, A. Banchs, J. Herzen, and P. Thiran, "Performance Analysis of MAC for Power-line Communications," in ACM Sigmetrics, 2014.

[8] C. Cano and D. Malone, "On Efficiency and Validity of Previous Homeplug MAC Performance Analysis," preprint arXiv:1401.6803, 2014

[9] C. Vlachou, J. Herzen, and P. Thiran, "Fairness of MAC protocols: IEEE 1901 vs. 802.11," in Power Line Communications and Its Applications (ISPLC), 2013 17th IEEE International Symposium on, 2013, pp. 58-63.

[10] A. Kumar, E. Altman, D. Miorandi, and M. Goyal, "New Insights From a Fixed-Point Analysis of Single Cell IEEE 802.11 WLANs," Networking, IEEE/ACM Transactions on, vol. 15, no. 3, pp. 588-601, june 2007.

[11] J.-w. Cho, J.-Y. Le Boudec, and Y. Jiang, "On the asymptotic validity of the decoupling assumption for analyzing 802.11 MAC protocol,' Information Theory, IEEE Transactions on, vol. 58, no. 11, 2012.

[12] K. Huang, K. Duffy, and D. Malone, "On the Validity of IEEE 802.11 MAC Modeling Hypotheses," Networking, IEEE/ACM Transactions on, vol. 18, no. 6, pp. 1935-1948, 2010.

[13] G. Sharma, A. Ganesh, and P. Key, "Performance Analysis of Contention Based Medium Access Control Protocols," Information Theory, IEEE Transactions on, vol. 55, no. 4, pp. 1665 -1682, april 2009.

[14] G. Berger-Sabbatel, A. Duda, O. Gaudoin, M. Heusse, and F. Rousseau, "Fairness and its impact on delay in 802.11 networks," in IEEE GLOBECOM '04, vol. 5, nov.-3 dec. 2004, pp. 2967-2973 Vol.5.

[15] M. Bredel and M. Fidler, "Understanding Fairness and its Impact on Quality of Service in IEEE 802.11," in IEEE INFOCOM 2009.

[16] C. Vlachou, A. Banchs, J. Herzen, and P. Thiran. On the MAC for Power-Line Communications: Modeling Assumptions and Performance Tradeoffs, Technical Report, 2014. Infoscience EPFL.

[17] C. Vlachou, J. Herzen, and P. Thiran. Simulator and Experimental Framework for the MAC of Power-Line Communications, Technical Report, 2014. Infoscience EPFL.

[18] OpenWrt. http://openwrt.org/.

[19] Atheros Open Powerline Toolkit. https://github.com/qca/open-plc-utils.

[20] H. W. Jain R., Chiu D.M., "A quantitative measure of fairness and discrimination for resource allocation in shared computer systems," $D E C$ Research Report TR-301, 1984.

[21] Z.-H. Sun. (2013) Inequalities for binomial coefficients. http://arxiv.org/ abs/1310.0353. 